\title{
COMPARATIVE STUDY OF BRACING SYSTEMS FOR IRREGULAR TALL STEEL STRUCTURES BASED IN DIFFERENT CITIES
}

\author{
Karthik K Vidyaranya ${ }^{1}$, Shiva Kumar $\mathrm{KS}^{2}$ \\ ${ }^{1}$ BE Student, Department of Civil Engineering, Global Academy of Technology, Karnataka, India \\ ${ }^{2}$ Assistant Professor, Department of Civil Engineering, Global Academy of Technology, Karnataka, India
}

\begin{abstract}
In a high-rise steel building, bracing system is an essential commodity for inadequate lateral reinforcements in the structure. Therefore, bracing system is a necessary solution for these lateral deflections caused by lateral loads. The resistance to wind and seismic load is one of the factor for the sustainability of the high rise building. For present study, plan irregularity with $(A / L)$ ratio of $(40 / 65)=0.62$ is considered as per IS 1893 (Part 1): 2002. In this study, the steel structure is assumed to be at 4 different cities with different seismic zones those cities are Agra, Bengaluru, Mumbai and Roorkee with five types of bracing systems. All cities are compared with an unbraced model of Roorkee city. A G+14 structure is analyzed for seismic zone II, zone III and zone IV as per IS: 1893-2002 using STAAD pro software. Dead and live loads are sustained by beams and columns whereas; the lateral loads are carried by bracing systems only. The bracings are provided at every corner of the structure. The structure is studied for nodal displacements in $X$-direction and $Z$ direction in all the floors, axial force and bending moment for the ground level column is compared and finally, drift index and corresponding percentage change in top roof is calculated.
\end{abstract}

Keywords: Lateral Loads, Bracing Systems, Axial Force, Bending Moment, Drift Index.

\section{INTRODUCTION}

Tall structures are subjected to high intensity wind load, and the displacement increases with increase in height. Seismic zones play an important role in the variation of these displacements in the structure. Due to repeated swaying of the structure by the lateral forces the resulting swaying motion increases the discomfort of the occupants and also it will increase the possibility of failure of the structure. As far as the ultimate limit state is concerned, lateral deflections must be limited to prevent second order p-delta effect due to gravity loading being of such a magnitude which may be sufficient to precipitate collapse. The strength and serviceability of the structure depends upon lateral stiffness and is a major consideration in the design of tall structure. The simple parameter that is used to estimate the lateral stiffness of a building is the drift index defined as the ratio of the maximum deflections at the top of the building to the total height.

Different bracing systems are used to improve lateral stiffness and reduce the drift index of the tall structure. In this study, different arrangements of bracing system are compared with various cities, in another word different lateral load condition. Bracing is a highly efficient and economical method to increase the lateral stiffness of the framed structures against lateral loads. Bracing systems helps in reducing the sizes of columns and beams thus, reducing the economy of the building.

\section{BRACING SYSTEM}

A bracing system is a structural system which is designed primarily to resist wind and seismic forces. Braced frames are designed to work in tension and compression similar to a truss. Braced-frames virtually eliminate the columns and girder bending factors and thus improve the efficiency of the pure rigid frame actions.

There are two types of bracing systems.

1) Concentric bracing system

2) Eccentric bracing system

1) Concentric bracing- These are the type of bracings whose centroid axis coincides with each other. They mainly increase the lateral stiffness of the frame which in turn increases the natural frequency and also decreases the lateral storey drift. Further, the bracing increases the axial compression in the columns to which they are connected by decreasing the bending moments and shear forces in the column.

2) Eccentric bracing- These are the type of bracing whose centerline braces are offset from the intersection of the centerline of columns and beams. It mainly improves the energy dissipation capacity and reduces the lateral stiffness of the system. At the point of connection of eccentric bracings on the beams, the vertical component of the bracing force due to earthquake causes concentrated load.

\section{OBJECTIVE}

The objective of this study is to compare the effects of lateral loads on a tall structure subjected to basic loads and lateral loads, hypothetically located in 4 cities namely Agra, Bengaluru, Mumbai and Roorkee. 5 types of bracing arrangements are made and the displacements, axial force, bending moment and drift index are compared. 


\section{MODELING AND ANALYSIS}

In the present study a three dimensional L shaped framed structure with $65 \mathrm{~m} * 65 \mathrm{~m}$ plan size and 15 numbers of stories is selected for the study. Storey height of $3 \mathrm{~m}$ with 13 bays of $5 \mathrm{~m}$ each along $\mathrm{X}$ and $\mathrm{Z}$ direction is provided for structure. The columns and beams are designed to withstand the live and dead loads adequately. The bracing sections are provided at the corners of the whole section. The lateral loads to be applied on the building are based on the Indian standards. The study is performed for seismic zone II, III, IV as per IS-1893 (Part1):2002 and wind speed of 47, 33, 44 and $39 \mathrm{~m} / \mathrm{s}$ as per IS-875:1987 for the cities Agra, Bengaluru, Mumbai and Roorkee respectively. The frames are assumed to be firmly fixed and the soil structure interaction is neglected. The load combinations are taken as per IS codes.

Five major bracing systems are analyzed with an un-braced reference model structure. They are as follows:
I. Diagonal bracing system
II. Eccentric bracing system
III. Knee bracing system
IV. V bracing system
V. X bracing system

\subsection{Data Considered for Analysis}

Table 1. Basic modeling data

\begin{tabular}{|l|l|}
\hline Type of structure & Steel moment resisting frame \\
\hline Number of stories & 15 \\
\hline Height of each storey & 3 \\
\hline Type of building & Industrial \\
\hline
\end{tabular}

\subsection{Specification based on Cities}

\subsubsection{Agra}

Table 2. Seismic zone and wind speed

\begin{tabular}{|l|l|}
\hline Seismic zone & III \\
\hline Basic wind speed & $47 \mathrm{~m} / \mathrm{s}$ \\
\hline
\end{tabular}

\subsubsection{Bengaluru}

Table 3. Seismic zone and wind speed

\begin{tabular}{|l|l|}
\hline Seismic zone & II \\
\hline Basic wind speed & $33 \mathrm{~m} / \mathrm{s}$ \\
\hline
\end{tabular}

\subsubsection{Mumbai}

Table 4. Seismic zone and wind speed

\begin{tabular}{|l|l|}
\hline Seismic zone & III \\
\hline Basic wind speed & $44 \mathrm{~m} / \mathrm{s}$ \\
\hline
\end{tabular}

\subsubsection{Roorkee}

Table 5. Seismic zone and wind speed

\begin{tabular}{|l|l|}
\hline Seismic zone & IV \\
\hline Basic wind speed & $39 \mathrm{~m} / \mathrm{s}$ \\
\hline
\end{tabular}

An I section of ISMB 200 @ $25.4 \mathrm{Kg} / \mathrm{m}$ is used throughout the structure as a beam member. To withstand the dead and live loads, a column of ISMB $300 @ 44.2 \mathrm{Kg} / \mathrm{m}$ is chosen. The channel section used for present study is ISMC 150 @ $16.4 \mathrm{Kg} / \mathrm{m}$.

Table 6. Loads considered for analysis

\begin{tabular}{|l|l|}
\hline Dead load on slab & $4 \mathrm{kN} / \mathrm{m}^{2}$ \\
\hline Live load on slab & $4 \mathrm{kN} / \mathrm{m}^{2}$ \\
\hline Wall load on beams & $10 \mathrm{kN} / \mathrm{m}$ \\
\hline
\end{tabular}

Table 7. Earthquake load parameter for Agra

\begin{tabular}{|l|l|}
\hline Zone factor, Z & 0.16 for zone III \\
\hline Importance factor, I & 1.0 \\
\hline Type of soil & II (medium) \\
\hline Response reduction factor, R & 5 \\
\hline $\begin{array}{l}\text { Percentage of imposed load } \\
\text { considered during seismic load } \\
\text { calculations }\end{array}$ & $50 \%$ \\
\hline
\end{tabular}

Table 8. Earthquake load parameter for Bengaluru

\begin{tabular}{|l|l|}
\hline Zone factor, Z & 0.1 for zone II \\
\hline Importance factor, I & 1.0 \\
\hline Type of soil & II (medium) \\
\hline Response reduction factor, R & 5 \\
\hline $\begin{array}{l}\text { Percentage of imposed load } \\
\text { considered during seismic load } \\
\text { calculations }\end{array}$ & $50 \%$ \\
\hline
\end{tabular}

Table 9. Earthquake load parameter for Mumbai

\begin{tabular}{|l|l|}
\hline Zone factor, Z & 0.16 for zone III \\
\hline Importance factor, I & 1.0 \\
\hline Type of soil & II (medium) \\
\hline Response reduction factor, R & 5 \\
\hline $\begin{array}{l}\text { Percentage of imposed load } \\
\text { considered during seismic load } \\
\text { calculations }\end{array}$ & $50 \%$ \\
\hline
\end{tabular}

Table 10. Earthquake load parameter for Roorkee

\begin{tabular}{|l|l|}
\hline Zone factor, Z & 0.24 for zone IV \\
\hline Importance factor, I & 1.0 \\
\hline Type of soil & II (medium) \\
\hline Response reduction factor, R & 5 \\
\hline $\begin{array}{l}\text { Percentage of imposed load } \\
\text { considered during seismic load } \\
\text { calculations }\end{array}$ & $50 \%$ \\
\hline
\end{tabular}

Table 11. Wind load parameters for Agra

\begin{tabular}{|l|l|}
\hline Basic wind speed, $\mathrm{V}_{\mathrm{b}}$ & $47 \mathrm{~m} / \mathrm{s}$ \\
\hline Risk co-efficient factor, $\mathrm{k} 1$ & 1 \\
\hline $\begin{array}{l}\text { Terrain, height and structure size } \\
\text { factor, } \mathrm{k} 2\end{array}$ & 1 \\
\hline Topography factor, $\mathrm{k} 3$ & 1 \\
\hline Class of structure & $\mathrm{B}$ \\
\hline
\end{tabular}


Table 12. Wind load parameters for Bengaluru

\begin{tabular}{|l|l|}
\hline Basic wind speed, $\mathrm{V}_{\mathrm{b}}$ & $33 \mathrm{~m} / \mathrm{s}$ \\
\hline Risk co-efficient factor, $\mathrm{k} 1$ & 1 \\
\hline Terrain, height and structure size factor, $\mathrm{k} 2$ & 1 \\
\hline Topography factor, $\mathrm{k} 3$ & 1 \\
\hline Class of structure & $\mathrm{C}$ \\
\hline
\end{tabular}

Table 13. Wind load parameters for Mumbai

\begin{tabular}{|l|l|}
\hline Basic wind speed, $\mathrm{V}_{\mathrm{b}}$ & $44 \mathrm{~m} / \mathrm{s}$ \\
\hline Risk co-efficient factor, $\mathrm{k} 1$ & 1 \\
\hline Terrain, height and structure size factor, $\mathrm{k} 2$ & 1 \\
\hline Topography factor, $\mathrm{k} 3$ & 1 \\
\hline Class of structure & $\mathrm{C}$ \\
\hline
\end{tabular}

Table 14. Wind load parameters for Roorkee

\begin{tabular}{|l|l|}
\hline Basic wind speed, $\mathrm{V}_{\mathrm{b}}$ & $39 \mathrm{~m} / \mathrm{s}$ \\
\hline Risk co-efficient factor, $\mathrm{k} 1$ & 1 \\
\hline Terrain, height and structure size factor, $\mathrm{k} 2$ & 1 \\
\hline Topography factor, $\mathrm{k} 3$ & 1 \\
\hline Class of structure & $\mathrm{C}$ \\
\hline
\end{tabular}

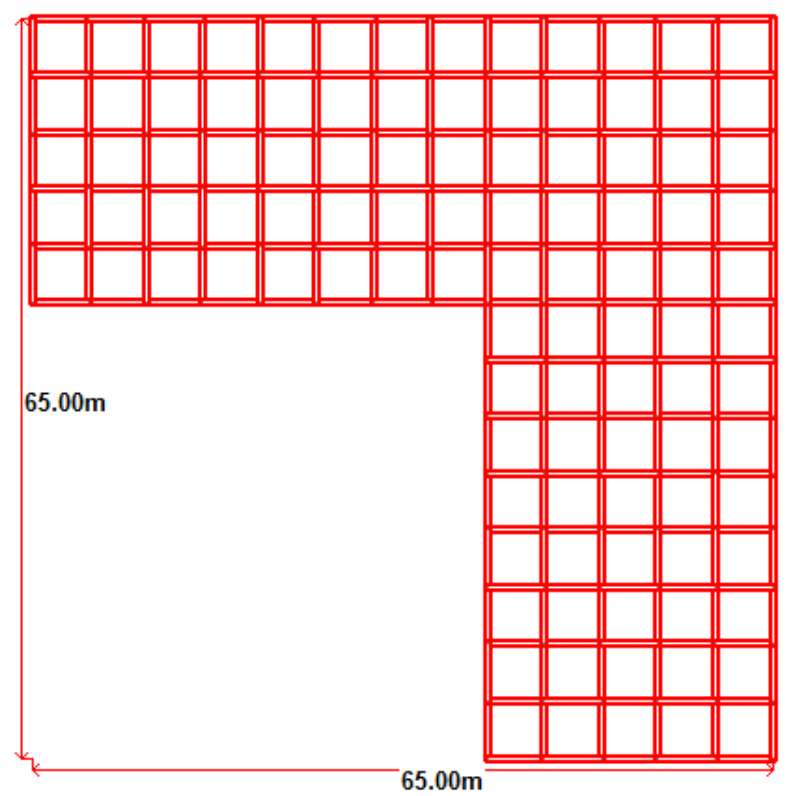

Fig.1 Plan un-braced reference model



Fig.2 Elevation of un-braced reference model



Fig.3 Elevation of Diagonal bracing system

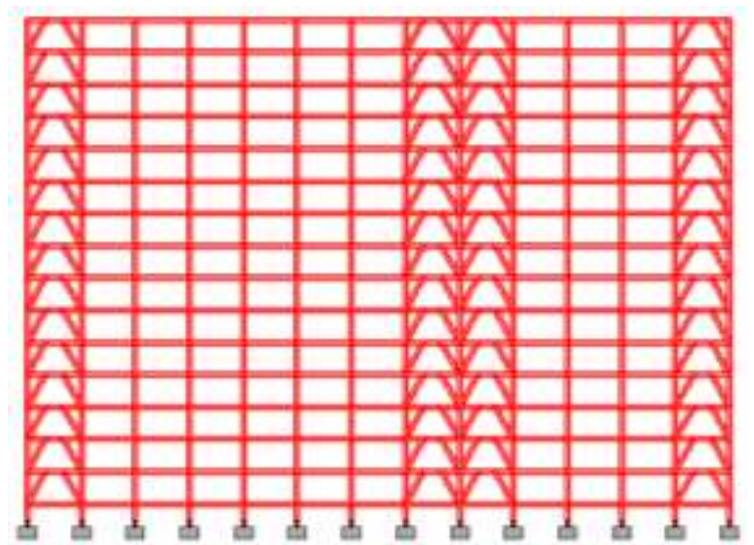

Fig.4 Elevation of Eccentric bracing system

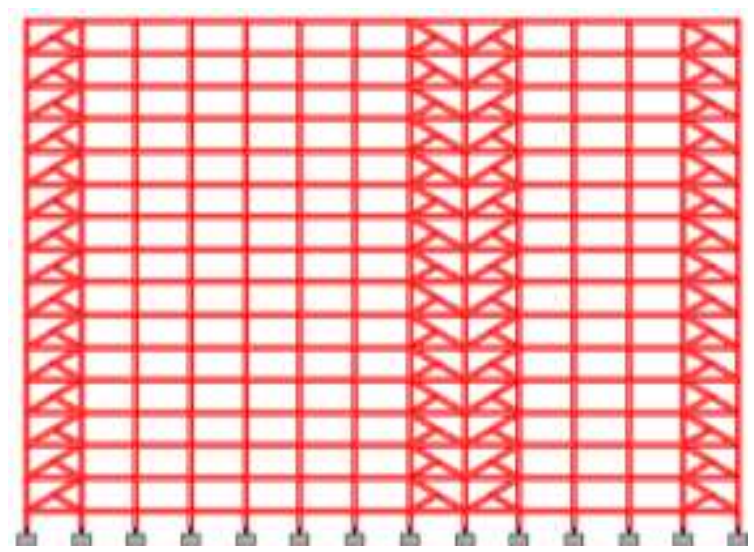

Fig.5 Elevation of Knee bracing system

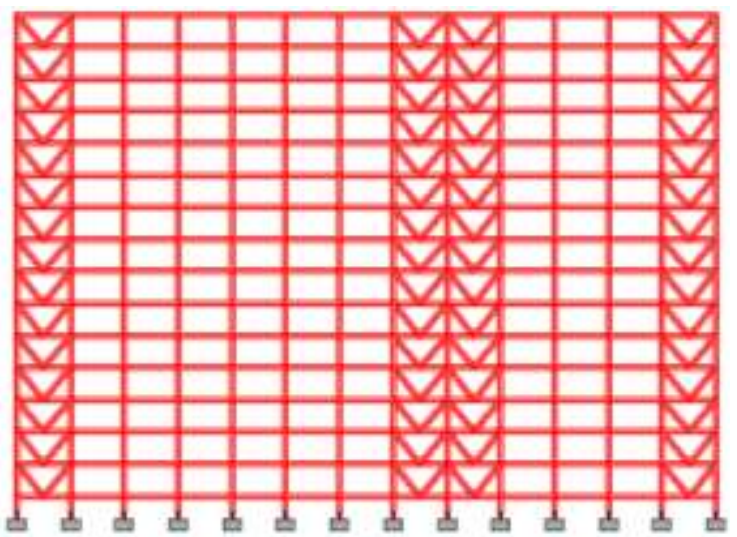

Fig.6 Elevation of V bracing system 


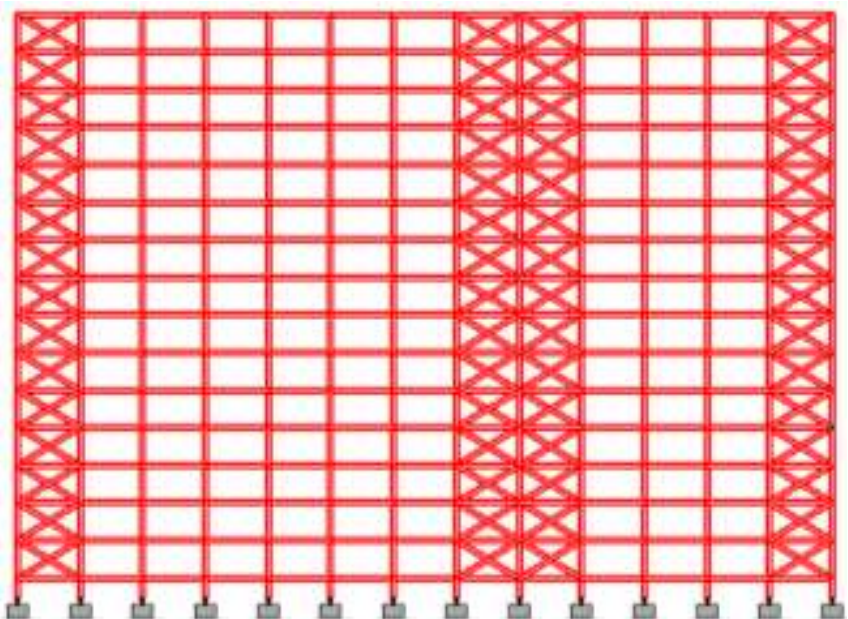

Fig.7 Elevation of $\mathrm{X}$ bracing system

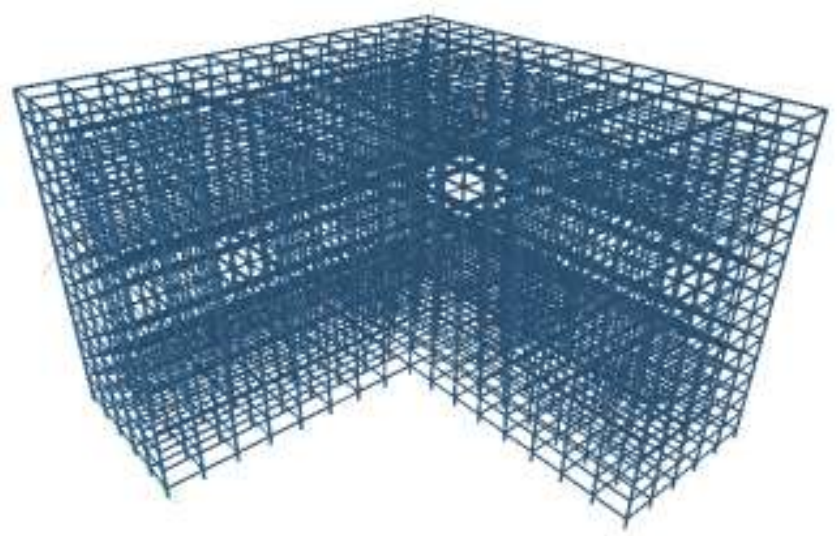

Fig.8 3D view of un-braced reference model

\section{RESULTS}

Table 15. Displacement in Diagonal bracing system in $\mathrm{mm}$, X-DIRECTION

\begin{tabular}{|r|r|r|r|r|r|}
\hline HEIGHT(m) & REFERENCE & \multicolumn{1}{l}{ AGRA } & \multicolumn{1}{l|}{ BLR } & MUMBAI & ROORKEE \\
\hline 0 & 0 & 0 & 0 & 0 & 0 \\
\hline 1.5 & 8.674 & 9.931 & 4.796 & 7.461 & 4.302 \\
\hline 4.5 & 59.241 & 51.806 & 23.833 & 38.105 & 57.134 \\
\hline 7.5 & 127.445 & 111.454 & 51.316 & 81.993 & 122.911 \\
\hline 10.5 & 196.097 & 171.555 & 78.932 & 126.19 & 189.202 \\
\hline 13.5 & 265.335 & 232.185 & 106.817 & 170.78 & 256.065 \\
\hline 16.5 & 333.794 & 292.17 & 134.412 & 214.901 & 322.219 \\
\hline 19.5 & 400.392 & 350.567 & 161.275 & 257.853 & 386.623 \\
\hline 22.5 & 464.168 & 406.529 & 187.016 & 299.012 & 448.341 \\
\hline 25.5 & 524.112 & 459.162 & 211.226 & 337.724 & 506.388 \\
\hline 28.5 & 579.142 & 507.504 & 233.462 & 373.279 & 559.703 \\
\hline 31.5 & 628.107 & 550.528 & 253.251 & 404.923 & 607.152 \\
\hline 34.5 & 669.844 & 587.189 & 270.113 & 431.887 & 647.584 \\
\hline 37.5 & 703.298 & 616.553 & 283.625 & 453.486 & 679.968 \\
\hline 40.5 & 727.895 & 638.069 & 293.544 & 469.322 & 703.692 \\
\hline 43.5 & 744.052 & 652.282 & 300.177 & 479.823 & 719.35 \\
\hline 46.5 & 753.632 & 660.092 & 303.81 & 485.587 & 727.956 \\
\hline
\end{tabular}

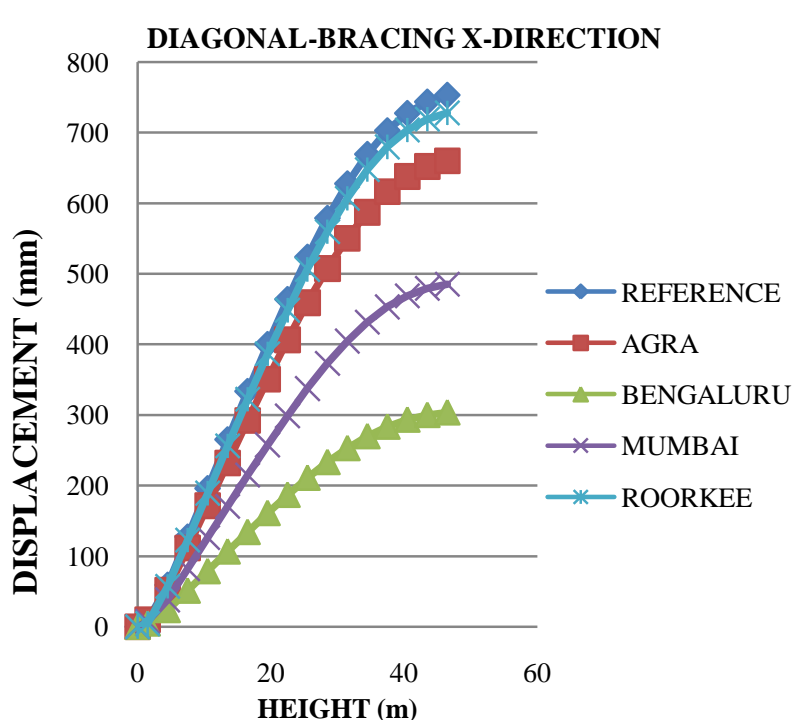

Fig.9 Displacement vs Height of Diagonal bracing system in $\mathrm{X}$-direction

Table 16. Displacement in Eccentric bracing system in mm, X-DIRECTION

\begin{tabular}{|r|r|r|r|r|r|}
\hline HEIGHT $(\mathrm{m})$ & REFERENCE & AGRA & BLR & MUMBAI & ROORKEE \\
\hline 0 & 0 & 0 & 0 & 0 & 0 \\
\hline 1.5 & 8.674 & 7.902 & 4.974 & 7.902 & 11.806 \\
\hline 4.5 & 59.241 & 64.053 & 34.787 & 56.742 & 77.866 \\
\hline 7.5 & 127.445 & 111.794 & 69.967 & 111.794 & 167.587 \\
\hline 10.5 & 196.097 & 172.133 & 107.658 & 172.133 & 258.1 \\
\hline 13.5 & 265.335 & 233.133 & 145.805 & 233.133 & 349.572 \\
\hline 16.5 & 333.794 & 293.657 & 183.657 & 293.657 & 440.325 \\
\hline 19.5 & 400.392 & 352.835 & 220.661 & 352.835 & 529.06 \\
\hline 22.5 & 464.168 & 409.9 & 257.794 & 409.9 & 615.412 \\
\hline 25.5 & 524.112 & 464.069 & 290.222 & 464.069 & 695.867 \\
\hline 28.5 & 579.142 & 514.49 & 321.75 & 514.49 & 771.476 \\
\hline 31.5 & 628.107 & 560.248 & 350.363 & 560.248 & 840.095 \\
\hline 34.5 & 669.844 & 600.4 & 375.469 & 600.4 & 900.309 \\
\hline 37.5 & 703.298 & 634.062 & 396.52 & 634.062 & 950.786 \\
\hline 40.5 & 727.895 & 660.618 & 413.146 & 660.618 & 990.58 \\
\hline 43.5 & 744.052 & 680.021 & 425.288 & 680.021 & 1019.664 \\
\hline 46.5 & 753.632 & 692.317 & 433.746 & 692.317 & 1039.125 \\
\hline
\end{tabular}



Fig.10 Displacement vs Height of Eccentric bracing system in X-direction 
Table 17. Displacement in Knee bracing system in mm, XDIRECTION

\begin{tabular}{|r|r|r|r|r|r|}
\hline HEIGHT $(\mathrm{m})$ & REFERENCE & AGRA & BLR & MUMBAI & ROORKEE \\
\hline 0 & 0 & 0 & 0 & 0 & 0 \\
\hline 1.5 & 8.674 & 10.299 & 6.884 & 10.299 & 14.845 \\
\hline 4.5 & 59.241 & 51.378 & 32.136 & 51.378 & 77.034 \\
\hline 7.5 & 127.445 & 110.613 & 69.235 & 110.613 & 165.806 \\
\hline 10.5 & 196.097 & 170.323 & 106.536 & 170.323 & 255.372 \\
\hline 13.5 & 265.335 & 230.698 & 144.296 & 230.698 & 345.903 \\
\hline 16.5 & 333.794 & 290.614 & 181.77 & 290.614 & 435.738 \\
\hline 19.5 & 400.392 & 349.207 & 218.414 & 349.207 & 523.597 \\
\hline 22.5 & 464.168 & 405.727 & 253.762 & 405.727 & 608.348 \\
\hline 25.5 & 524.112 & 459.39 & 287.321 & 459.39 & 688.814 \\
\hline 28.5 & 579.142 & 509.35 & 318.565 & 509.35 & 763.731 \\
\hline 31.5 & 628.107 & 554.701 & 364.926 & 554.701 & 831.726 \\
\hline 34.5 & 669.844 & 594.502 & 371.814 & 594.502 & 891.42 \\
\hline 37.5 & 703.298 & 627.873 & 392.684 & 627.873 & 941.458 \\
\hline 40.5 & 727.895 & 654.197 & 409.167 & 654.197 & 980.903 \\
\hline 43.5 & 744.052 & 673.425 & 421.167 & 673.425 & 1009.723 \\
\hline 46.5 & 753.632 & 686.475 & 429.582 & 686.475 & 1028.998 \\
\hline & & & & & \\
\hline
\end{tabular}

\section{KNEE-BRACING X-DIRECTION}



Fig. 11 Displacement vs Height of Knee bracing system in $\mathrm{X}$-direction
Table 18. Displacement in V bracing system in $\mathrm{mm}, \mathrm{X}$ DIRECTION

\begin{tabular}{|r|r|r|r|r|r|}
\hline HEIGHT $(\mathrm{m})$ & REFERENCE & \multicolumn{1}{l|}{ AGRA } & llR & MUMBAI & ROORKEE \\
\hline 0 & 0 & 0 & 0 & 0 & 0 \\
\hline 1.5 & 8.674 & 52.689 & 28.662 & 46.282 & 40.019 \\
\hline 4.5 & 59.241 & 66.864 & 36.292 & 59.018 & 79.82 \\
\hline 7.5 & 127.445 & 110.321 & 69.068 & 110.321 & 165.347 \\
\hline 10.5 & 196.097 & 169.875 & 106.28 & 169.875 & 254.668 \\
\hline 13.5 & 265.335 & 230.095 & 143.951 & 230.095 & 346.786 \\
\hline 16.5 & 333.794 & 289.859 & 181.339 & 289.859 & 435.381 \\
\hline 19.5 & 400.392 & 348.306 & 217.9 & 348.306 & 522.182 \\
\hline 22.5 & 464.168 & 404.689 & 253.168 & 404.689 & 606.717 \\
\hline 25.5 & 524.112 & 458.223 & 286.654 & 458.223 & 686.983 \\
\hline 28.5 & 579.142 & 508.067 & 317.831 & 508.067 & 761.716 \\
\hline 31.5 & 628.107 & 553.314 & 346.131 & 553.314 & 829.558 \\
\hline 34.5 & 669.844 & 593.025 & 370.968 & 593.025 & 889.101 \\
\hline 37.5 & 703.298 & 626.321 & 391.795 & 626.321 & 939.022 \\
\hline 40.5 & 727.895 & 652.586 & 408.244 & 652.586 & 978.375 \\
\hline 43.5 & 744.052 & 671.77 & 420.253 & 671.77 & 1007.127 \\
\hline 46.5 & 753.632 & 684.79 & 428.617 & 684.79 & 1026.354 \\
\hline & & & & &
\end{tabular}

\section{V-BRACING X-DIRECTION}



HEIGHT (m)

Fig. 12 Displacement vs Height of V bracing system in Xdirection 
Table 19. Displacement in $\mathrm{X}$ bracing system in $\mathrm{mm}, \mathrm{X}$ DIRECTION

\begin{tabular}{|r|r|r|r|r|r|}
\hline HEIGHT(m) & REFERENCE & AGRA & BLR & MUMBAI & ROORKEE \\
\hline 0 & 0 & 0 & 0 & 0 & 0 \\
\hline 1.5 & 8.674 & 7.186 & 4.925 & 7.186 & 10.199 \\
\hline 4.5 & 59.241 & 37.936 & 23.718 & 37.936 & 56.893 \\
\hline 7.5 & 127.445 & 81.672 & 51.112 & 81.672 & 122.422 \\
\hline 10.5 & 196.097 & 125.634 & 78.552 & 125.634 & 188.409 \\
\hline 13.5 & 265.335 & 170.035 & 106.31 & 170.035 & 255.002 \\
\hline 16.5 & 333.794 & 213.97 & 133.774 & 213.97 & 320.892 \\
\hline 19.5 & 400.392 & 256.744 & 160.52 & 256.744 & 385.043 \\
\hline 22.5 & 464.168 & 297.738 & 186.148 & 297.738 & 446.524 \\
\hline 25.5 & 524.112 & 336.297 & 210.255 & 336.297 & 504.353 \\
\hline 28.5 & 579.142 & 371.715 & 232.397 & 371.715 & 557.471 \\
\hline 31.5 & 628.107 & 403.238 & 252.105 & 403.238 & 604.748 \\
\hline 34.5 & 669.844 & 430.097 & 268.869 & 430.097 & 645.031 \\
\hline 37.5 & 703.298 & 451.607 & 282.347 & 451.607 & 677.288 \\
\hline 40.5 & 727.895 & 467.393 & 292.237 & 467.393 & 700.933 \\
\hline 43.5 & 744.052 & 477.692 & 298.687 & 477.692 & 716.367 \\
\hline 46.5 & 753.632 & 483.935 & 302.811 & 483.935 & 725.434 \\
\hline & & & & & \\
\hline
\end{tabular}

X-BRACING X-DIRECTION

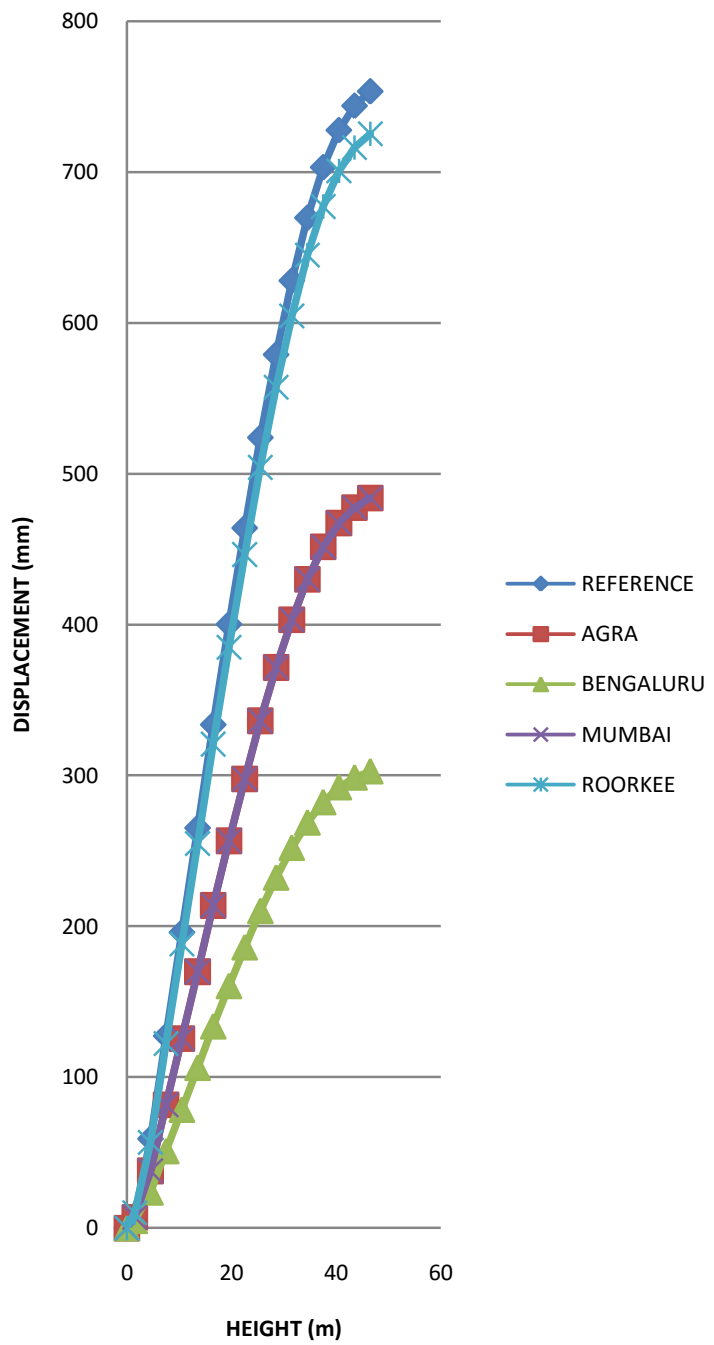

Fig. 13 Displacement vs Height of $\mathrm{X}$ bracing system in $\mathrm{X}$ direction
Table 20. Displacement in Diagonal bracing system in $\mathrm{mm}$, Z-DIRECTION

\begin{tabular}{|r|r|r|r|r|r|}
\hline HEIGHT(m) & REFERENCE & AGRA & BLR & MUMBAI & ROORKEE \\
\hline 0 & 0 & 0 & 0 & 0 & 0 \\
\hline 1.5 & 30.799 & 59.01 & 27.983 & 43.786 & 64.931 \\
\hline 4.5 & 233.057 & 177.561 & 81.783 & 130.649 & 195.805 \\
\hline 7.5 & 445.822 & 340.089 & 156.697 & 250.264 & 375.023 \\
\hline 10.5 & 586.493 & 448.022 & 206.353 & 329.653 & 494.054 \\
\hline 13.5 & 725.462 & 555.108 & 255.663 & 408.441 & 613.145 \\
\hline 16.5 & 861.879 & 660.878 & 304.365 & 486.259 & 728.785 \\
\hline 19.5 & 994.529 & 764.242 & 351.957 & 562.306 & 845.772 \\
\hline 22.5 & 1121.768 & 863.902 & 397.831 & 635.626 & 952.676 \\
\hline 25.5 & 1241.712 & 958.292 & 441.293 & 705.068 & 1056.769 \\
\hline 28.5 & 1352.215 & 1045.598 & 481.482 & 769.296 & 1153.049 \\
\hline 31.5 & 1450.855 & 1123.734 & 517.449 & 826.778 & 1239.217 \\
\hline 34.5 & 1534.943 & 1190.369 & 548.122 & 875.799 & 1312.702 \\
\hline 37.5 & 1601.522 & 1242.9 & 572.304 & 914.445 & 1370.633 \\
\hline 40.5 & 1647.474 & 1278.574 & 588.752 & 940.703 & 1409.97 \\
\hline 43.5 & 1670.65 & 1295.48 & 596.634 & 953.188 & 1428.594 \\
\hline 46.5 & 1675.555 & 1296.567 & 597.112 & 953.977 & 1429.796 \\
\hline
\end{tabular}

DIAGONAL-BRACING Z-DIRECTION



Fig. 14 Displacement vs Height of Diagonal bracing system in Z-direction 
Table 21. Displacement in Eccentric bracing system in $\mathrm{mm}$, Z-DIRECTION

\begin{tabular}{|r|r|r|r|r|r|}
\hline HEIGHT(m) & REFERENCE & AGRA & BLR & MUMBAI & ROORKEE \\
\hline 0 & 0 & 0 & 0 & 0 & 0 \\
\hline 1.5 & 30.799 & 48.767 & 30.714 & 48.767 & 72.837 \\
\hline 4.5 & 233.057 & 182.381 & 114.156 & 182.381 & 273.351 \\
\hline 7.5 & 445.822 & 349.347 & 218.717 & 349.347 & 523.523 \\
\hline 10.5 & 586.493 & 460.17 & 288.024 & 460.17 & 689.698 \\
\hline 13.5 & 725.462 & 570.251 & 356.915 & 570.251 & 854.699 \\
\hline 16.5 & 861.879 & 679.17 & 425.075 & 679.17 & 1017.964 \\
\hline 19.5 & 994.529 & 785.975 & 491.911 & 785.975 & 1178.061 \\
\hline 22.5 & 1121.768 & 889.522 & 556.704 & 889.522 & 1333.281 \\
\hline 25.5 & 1241.712 & 988.447 & 618.601 & 988.447 & 1481.574 \\
\hline 28.5 & 1352.215 & 1081.158 & 676.609 & 1081.158 & 1620.558 \\
\hline 31.5 & 1450.855 & 1165.84 & 729.589 & 1165.84 & 1747.508 \\
\hline 34.5 & 1534.943 & 1240.451 & 776.268 & 1240.451 & 1859.362 \\
\hline 37.5 & 1601.522 & 1302.724 & 815.226 & 1302.724 & 1952.721 \\
\hline 40.5 & 1647.474 & 1350.21 & 844.953 & 1350.21 & 2023.887 \\
\hline 43.5 & 1670.65 & 1380.614 & 864.037 & 1380.614 & 2069.383 \\
\hline 46.5 & 1675.555 & 1397.031 & 874.344 & 1397.031 & 2093.947 \\
\hline & & & & & \\
\hline
\end{tabular}

\section{ECCENTRIC-BRACING Z-DIRECTION}

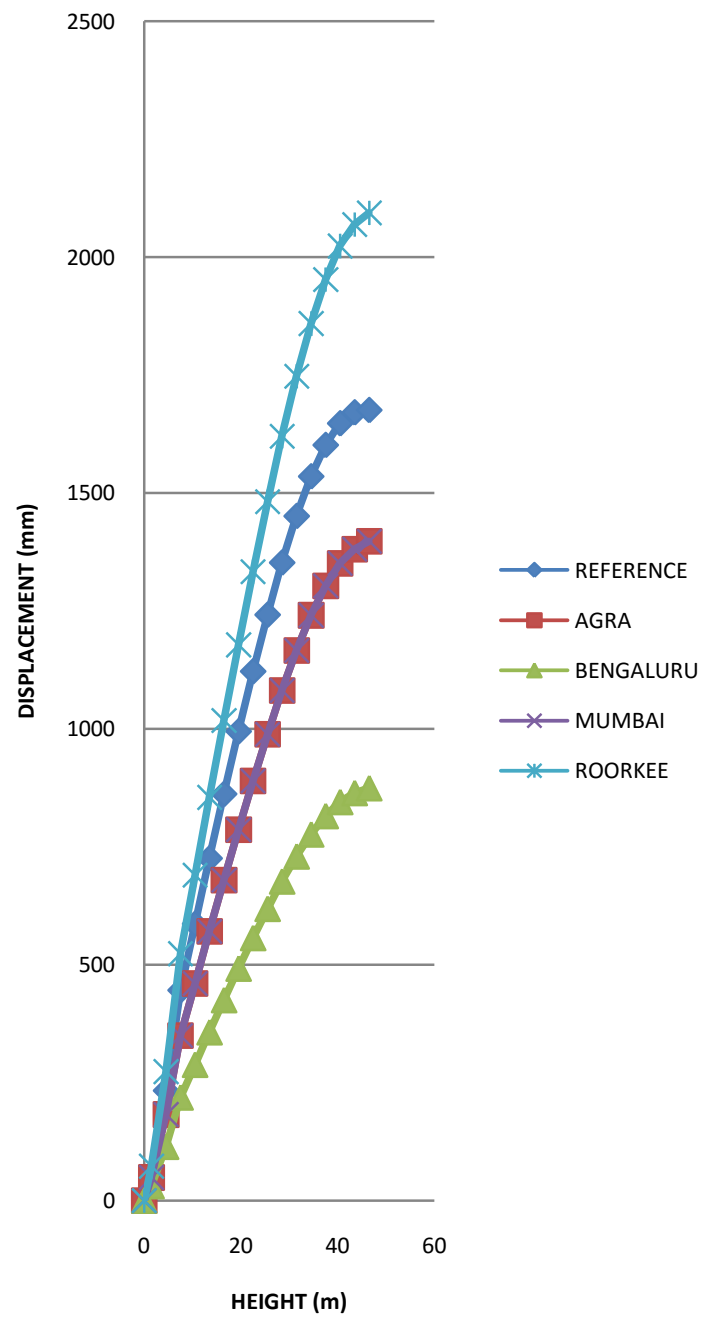

Fig. 15 Displacement vs Height of Eccentric bracing system in Z-direction
Table 22. Displacement in Knee bracing system in mm, ZDIRECTION

\begin{tabular}{|r|r|r|r|r|r|}
\hline HEIGHT(m) & REFERENCE & AGRA & BLR & MUMBA & ROORKEE \\
\hline 0 & 0 & 0 & 0 & 0 & 0 \\
\hline 1.5 & 30.799 & 58.207 & 37.482 & 58.207 & 85.842 \\
\hline 4.5 & 233.057 & 176.001 & 110.174 & 176.001 & 263.712 \\
\hline 7.5 & 445.822 & 337.222 & 211.15 & 337.222 & 505.318 \\
\hline 10.5 & 586.493 & 444.324 & 278.135 & 444.324 & 665.91 \\
\hline 13.5 & 725.462 & 550.83 & 344.794 & 550.83 & 825.544 \\
\hline 16.5 & 861.879 & 656.338 & 410.825 & 656.338 & 983.689 \\
\hline 19.5 & 994.529 & 759.927 & 475.652 & 759.927 & 1138.96 \\
\hline 22.5 & 1121.768 & 860.482 & 538.577 & 860.482 & 1289.691 \\
\hline 25.5 & 1241.712 & 956.666 & 598.762 & 956.666 & 1433.872 \\
\hline 28.5 & 1352.215 & 1046.912 & 655.229 & 1046.912 & 1569.157 \\
\hline 31.5 & 1450.855 & 1129.428 & 706.865 & 1129.428 & 1692.858 \\
\hline 34.5 & 1534.943 & 1202.194 & 752.381 & 1202.194 & 1801.944 \\
\hline 37.5 & 1601.522 & 1262.96 & 790.398 & 1262.96 & 1893.043 \\
\hline 40.5 & 1647.474 & 1309.755 & 819.404 & 1309.755 & 1962.481 \\
\hline 43.5 & 1670.65 & 1338.716 & 837.994 & 1338.716 & 2006.79 \\
\hline 46.5 & 1675.555 & 1354.712 & 847.919 & 1354.712 & 2030.437 \\
\hline & & & & & \\
\hline
\end{tabular}

KNEE-BRACING Z-DIRECTION

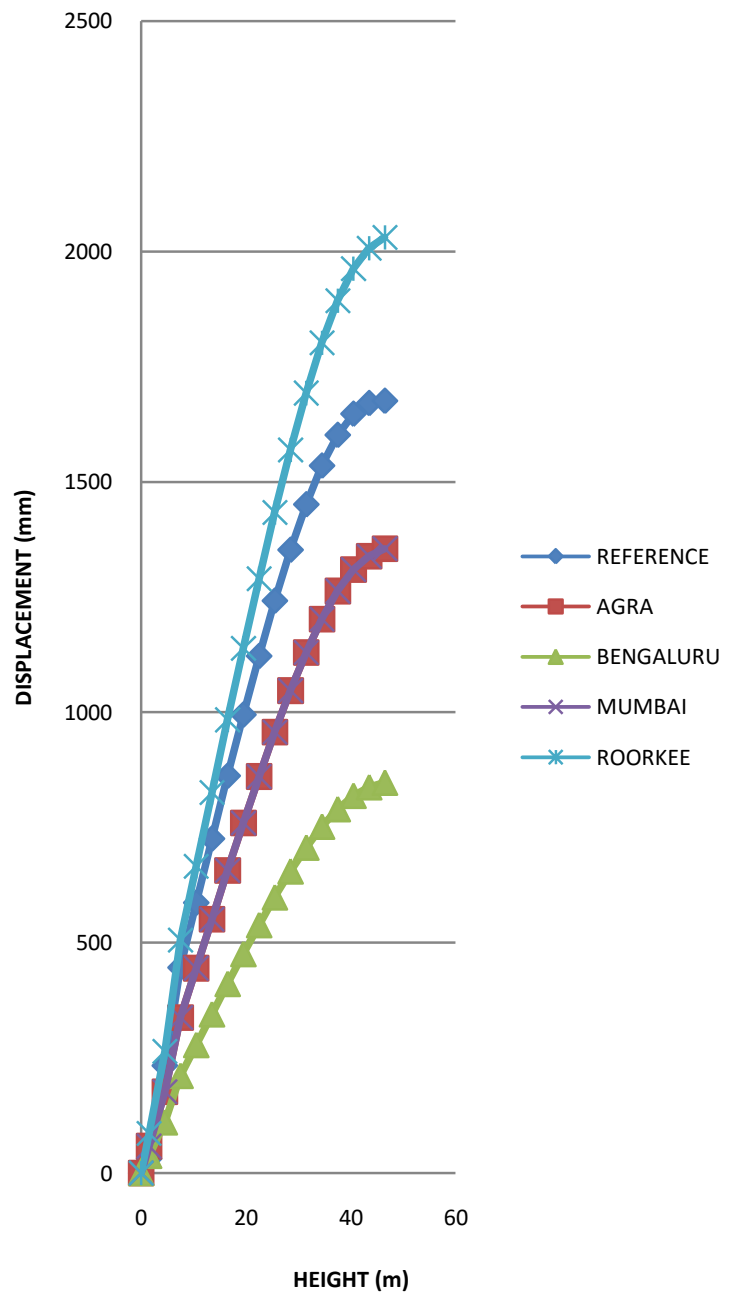

Fig. 16 Displacement vs Height of Knee bracing system in Z-direction 
Table 23. Displacement in V bracing system in $\mathrm{mm}, \mathrm{Z}$ DIRECTION

\begin{tabular}{|r|r|r|r|r|r|}
\hline HEIGHT $(\mathrm{m})$ & REFERENCE & lGRA & BLR & MUMBAI & ROORKEE \\
\hline 0 & 0 & 0 & 0 & 0 & 0 \\
\hline 1.5 & 30.799 & 67.047 & 35.99 & 59.111 & 84.341 \\
\hline 4.5 & 233.057 & 174.891 & 109.517 & 174.891 & 262.057 \\
\hline 7.5 & 445.822 & 335.109 & 209.901 & 335.109 & 502.054 \\
\hline 10.5 & 586.493 & 441.56 & 276.5 & 441.56 & 661.64 \\
\hline 13.5 & 725.462 & 547.44 & 342.789 & 547.44 & 820.367 \\
\hline 16.5 & 861.879 & 652.348 & 408.465 & 652.348 & 977.525 \\
\hline 19.5 & 994.529 & 755.369 & 472.957 & 755.369 & 1131.917 \\
\hline 22.5 & 1121.768 & 855.394 & 535.569 & 855.394 & 1281.829 \\
\hline 25.5 & 1241.712 & 951.031 & 595.466 & 951.031 & 1425.257 \\
\hline 28.5 & 1352.215 & 1040.896 & 651.673 & 1040.896 & 1559.861 \\
\hline 31.5 & 1450.855 & 1123.023 & 703.071 & 1123.023 & 1682.961 \\
\hline 34.5 & 1534.943 & 1195.457 & 748.4 & 1195.457 & 1791.533 \\
\hline 37.5 & 1601.522 & 1255.95 & 786.255 & 1255.95 & 1882.209 \\
\hline 40.5 & 1647.474 & 1302.076 & 815.816 & 1302.076 & 1951.325 \\
\hline 43.5 & 1670.65 & 1331.546 & 833.645 & 1331.546 & 1995.415 \\
\hline 46.5 & 1675.555 & 1347.248 & 843.509 & 1347.248 & 2018.901 \\
\hline
\end{tabular}

V-BRACING Z-DIRECTION

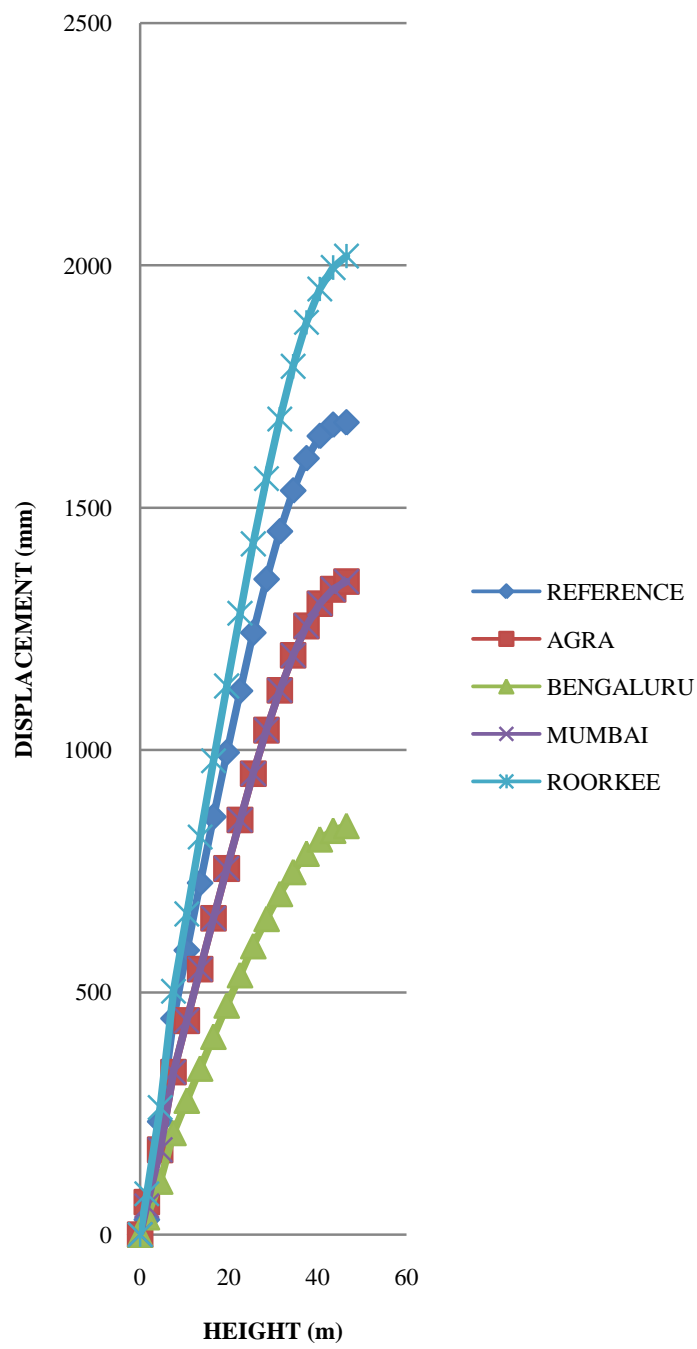

Fig. 17 Displacement vs Height of V bracing system in Zdirection
Table 24. Displacement in X bracing system in $\mathrm{mm}, \mathrm{Z}$ DIRECTION

\begin{tabular}{|r|r|r|r|r|r|}
\hline HEIGHT $(\mathrm{m})$ & REFERENCE & AGRA & BLR & MUMBAI & ROORKEE \\
\hline 0 & 0 & 0 & 0 & 0 & 0 \\
\hline 1.5 & 30.799 & 44.206 & 28.506 & 44.206 & 65.138 \\
\hline 4.5 & 233.057 & 129.178 & 80.884 & 129.178 & 193.569 \\
\hline 7.5 & 445.822 & 247.514 & 155.034 & 247.514 & 370.821 \\
\hline 10.5 & 586.493 & 325.992 & 204.914 & 325.992 & 488.499 \\
\hline 13.5 & 725.462 & 403.952 & 252.914 & 403.952 & 605.336 \\
\hline 16.5 & 861.879 & 480.974 & 301.125 & 480.974 & 720.773 \\
\hline 19.5 & 994.529 & 556.271 & 348.254 & 556.271 & 833.628 \\
\hline 22.5 & 1121.768 & 628.89 & 393.702 & 628.89 & 942.474 \\
\hline 25.5 & 1241.712 & 697.89 & 436.756 & 697.89 & 1045.599 \\
\hline 28.5 & 1352.215 & 761.335 & 476.584 & 761.335 & 1141.005 \\
\hline 31.5 & 1450.855 & 818.308 & 512.233 & 818.308 & 1226.408 \\
\hline 34.5 & 1534.943 & 866.891 & 542.632 & 866.891 & 1299.237 \\
\hline 37.5 & 1601.522 & 905.182 & 566.458 & 905.182 & 1356.636 \\
\hline 40.5 & 1647.474 & 931.172 & 582.875 & 931.172 & 1395.57 \\
\hline 43.5 & 1670.65 & 943.471 & 590.639 & 943.471 & 1413.914 \\
\hline 46.5 & 1675.555 & 944.261 & 591.174 & 944.261 & 1415.044 \\
\hline
\end{tabular}

\section{X-BRACING Z-DIRECTION}

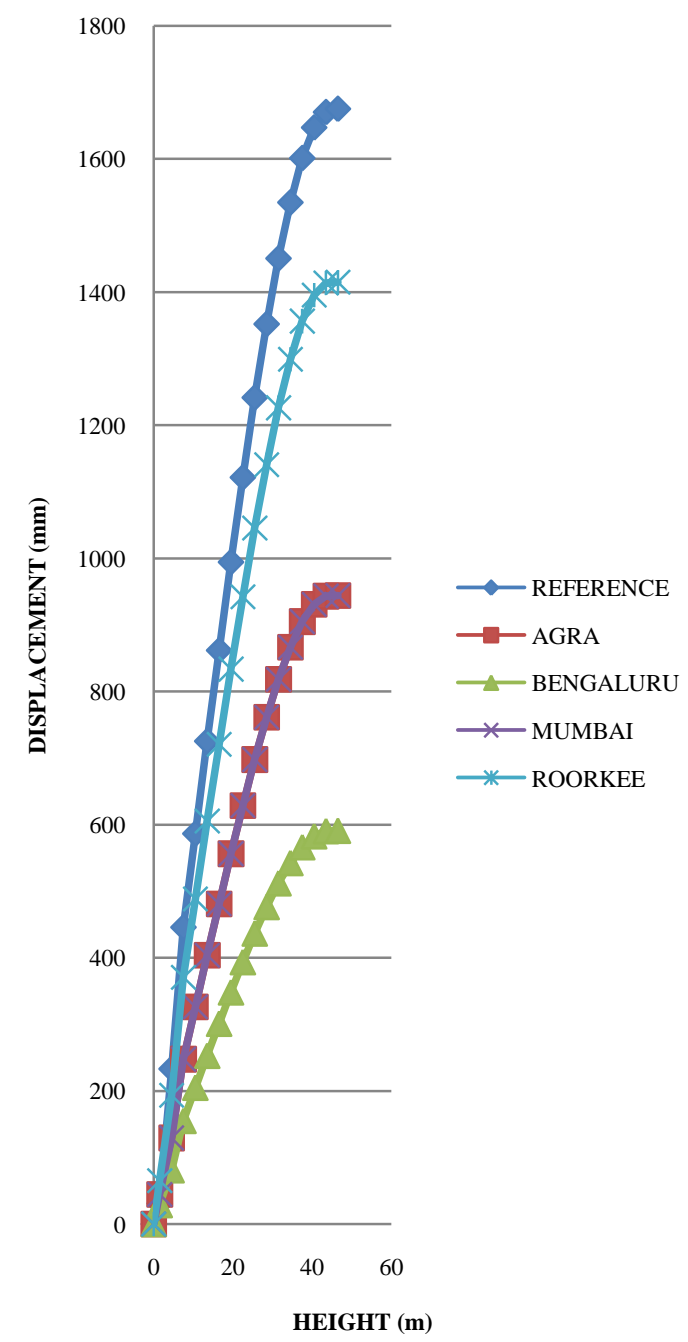

Fig. 18 Displacement vs Height of X bracing system in Zdirection 
Table 25. Maximum Axial force acting on ground level column for Diagonal bracing system in $\mathrm{kN}$

\begin{tabular}{|l|r|r|r|r|r|}
\hline & REFERENCE & AGRA & BLR & MUMBAI & ROORKEE \\
\hline AXIAL FORCE & 6890.345 & 5902.611 & 5257.41 & 5257.418 & 6307.907 \\
\hline
\end{tabular}

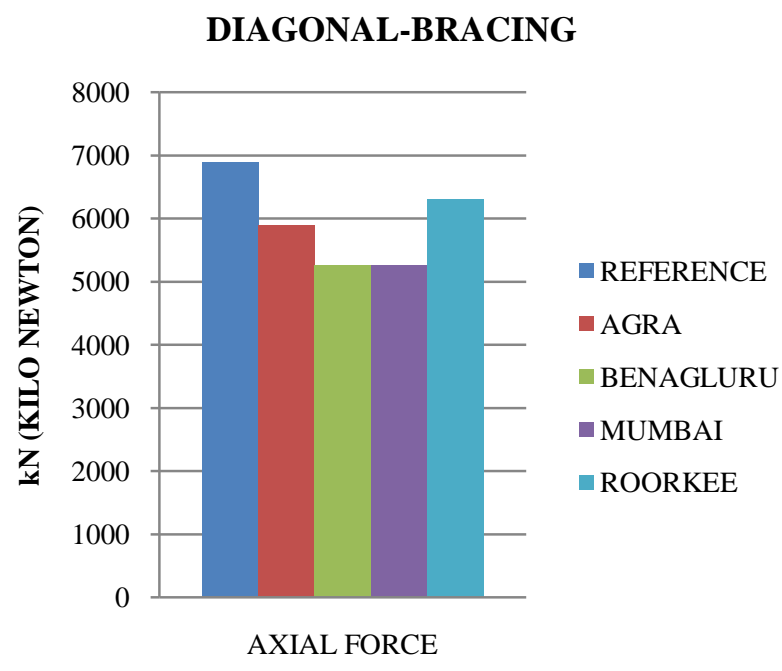

Fig. 19 Variation in Axial force for different cities in Diagonal bracing

Table 26. Maximum Axial force acting on ground level column for Eccentric bracing system in $\mathrm{kN}$

\begin{tabular}{|l|r|l|l|l|r|}
\hline & REFERENCE & AGRA & BLR & MUMBAI & ROORKEE \\
\hline AXIAL FORCE & 6890.345 & 6746.501 & 6746.501 & 6746.501 & 6746.501 \\
\hline
\end{tabular}

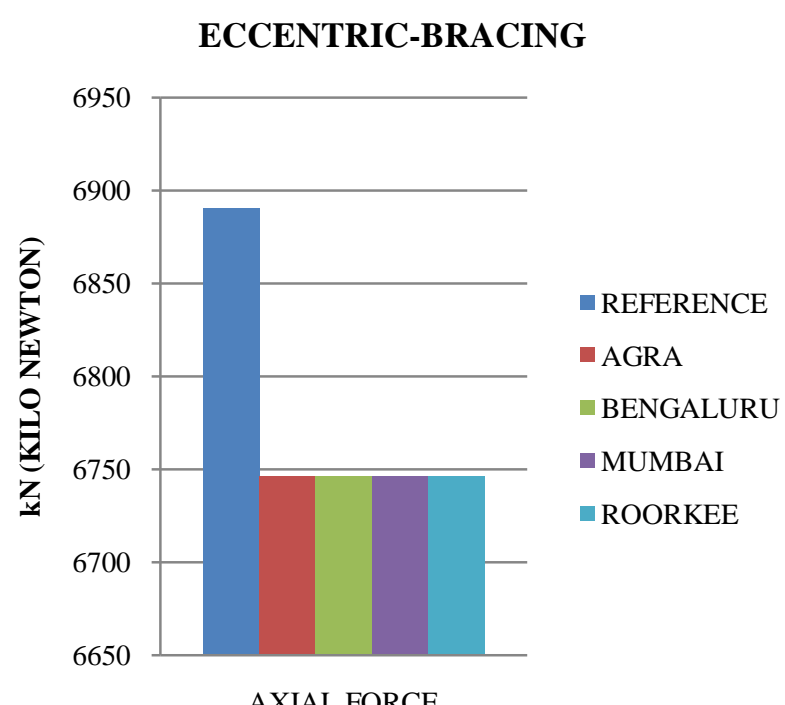

Fig. 20 Variation in Axial force for different cities in Eccentric bracing

Table 27. Maximum Axial force acting on ground level column for Knee bracing system in $\mathrm{kN}$

\begin{tabular}{|l|c|c|c|c|c|}
\hline & REFERENCE & AGRA & BLR & MUMBAI & ROORKEE \\
\hline AXIAL FORCE & 6890.345 & 7040.422 & 7040.422 & 7040.422 & 8108.624 \\
\hline
\end{tabular}

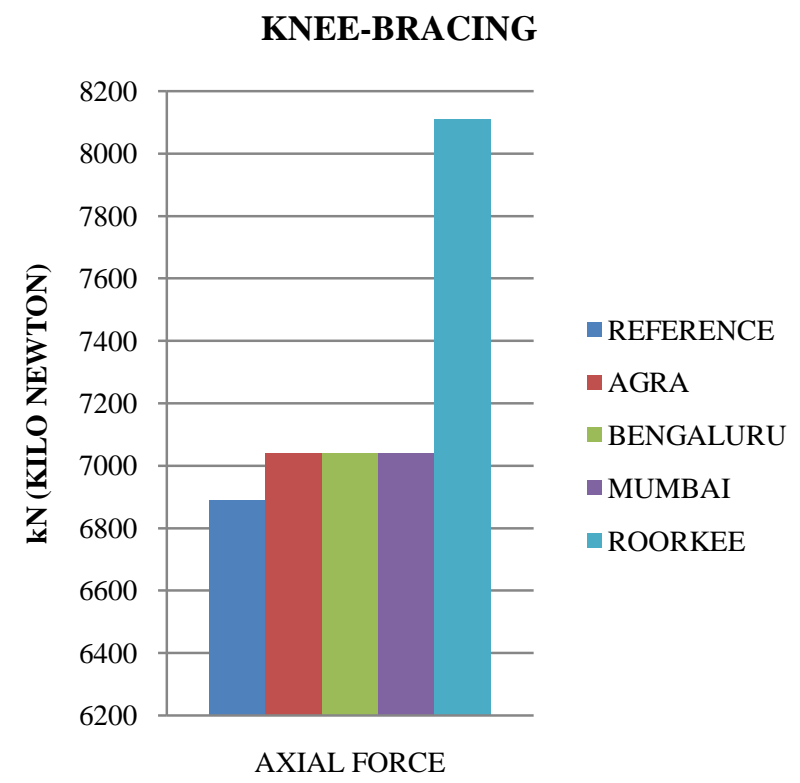

Fig. 21 Variation in Axial force for different cities in Knee bracing

Table 28. Maximum Axial force acting on ground level column for $\mathrm{V}$ bracing system in $\mathrm{kN}$

\begin{tabular}{|l|r|r|r|r|r|}
\hline & REFERENCE & AGRA & BLR & MUMBAI & ROORKEE \\
\hline AXIAL FORCE & 6890.345 & 6891.234 & 6891.234 & 6891.234 & 8144.901 \\
\hline
\end{tabular}

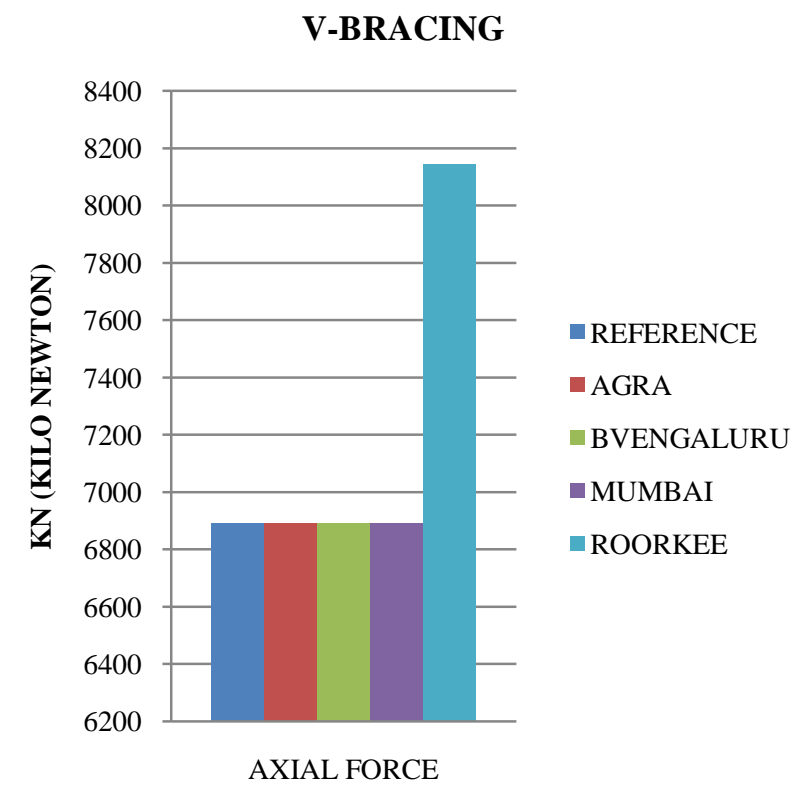

Fig. 22 Variation in Axial force for different cities in V bracing

Table 29. Maximum Axial force acting on ground level column for $\mathrm{X}$ bracing system in $\mathrm{kN}$

\begin{tabular}{|l|c|c|r|r|r|}
\hline & REFERENCE & AGRA & BLR & MUMBAI & ROORKEE \\
\hline AXIAL FORCE & 6890.345 & 7290.639 & 6960.796 & 7290.639 & 8833.08 \\
\hline
\end{tabular}






Fig. 23 Variation in Axial force for different cities in $\mathrm{X}$ bracing

Table 30. Maximum Bending moment acting on ground level column for Diagonal bracing system in $\mathrm{kN}-\mathrm{m}$ \begin{tabular}{|l|r|r|r|r|r|}
\hline & REFERENCE & AGRA & BLR & MUMBAI & ROORKEE \\
\hline BENDING MOMENT & 168.947 & 214.878 & 97.326 & 154.994 & 238.167 \\
\hline
\end{tabular}

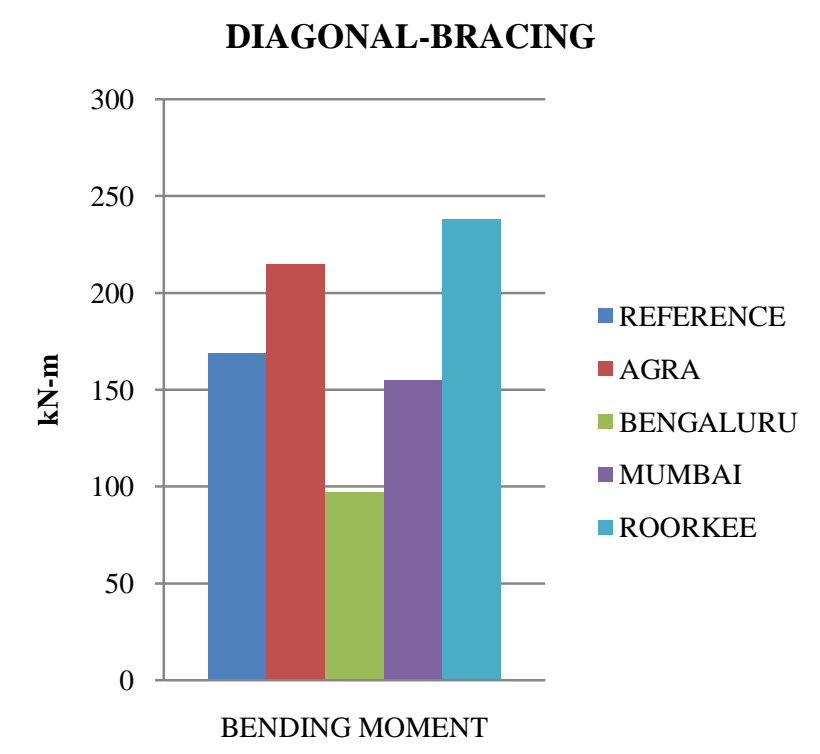

Fig. 24 Variation in Bending moment for different cities in Diagonal bracing

Table 31. Maximum Bending moment acting on ground level column for Eccentric bracing system in $\mathrm{kN}-\mathrm{m}$

\begin{tabular}{|l|r|r|r|r|r|}
\hline & REFERENCE & AGRA & BLR & MUMBAI & ROORKEE \\
\hline BENDING MOMENT & 168.947 & 162.045 & 101.349 & 162.045 & 243.374 \\
\hline
\end{tabular}

\section{ECCENTRIC-BRACING}

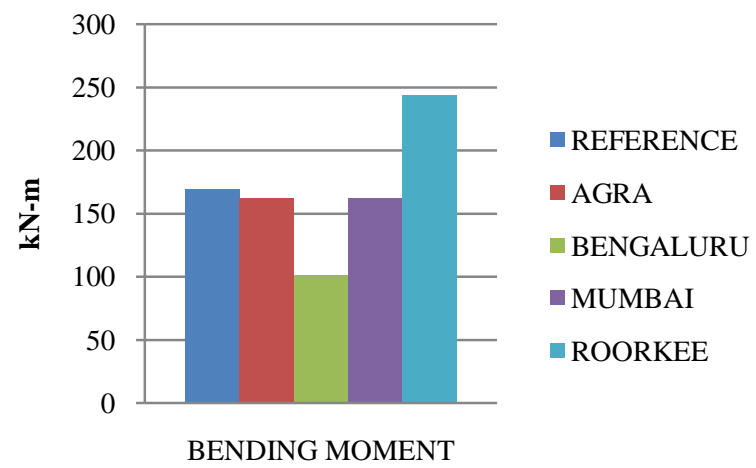

Fig. 25 Variation in Bending moment for different cities in Eccentric bracing

Table 32. Maximum Bending moment acting on ground level column for Knee bracing system in $\mathrm{kN}-\mathrm{m}$

\begin{tabular}{|l|c|c|c|r|r|}
\hline & REFERENCE & AGRA & BLR & MUMBAI & ROORKEE \\
\hline BENDING MOMENT & 168.947 & 200.203 & 137.003 & 200.203 & 299.274 \\
\hline
\end{tabular}

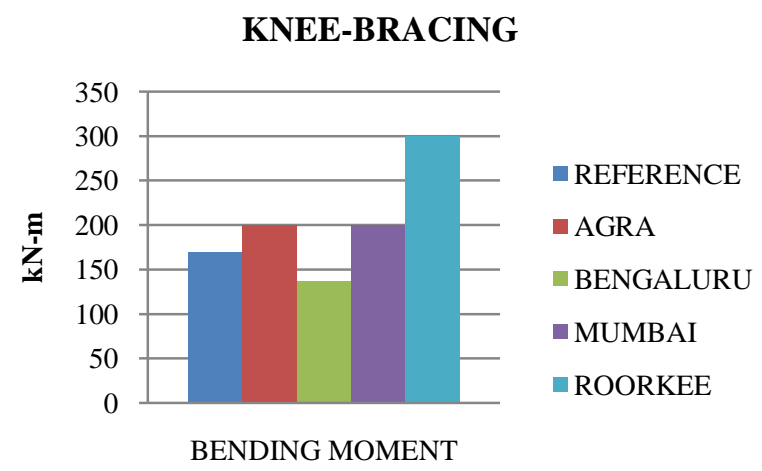

Fig. 26 Variation in Bending moment for different cities in Knee bracing

Table 33. Maximum Bending moment acting on ground level column for $\mathrm{V}$ bracing system in $\mathrm{kN}-\mathrm{m}$

\begin{tabular}{|l|r|r|r|r|r|}
\hline & REFERENCE & AGRA & BLR & MUMBAI & ROORKEE \\
\hline BENDING MOMENT & 168.947 & 187.661 & 115.85 & 187.661 & 283.409 \\
\hline
\end{tabular}

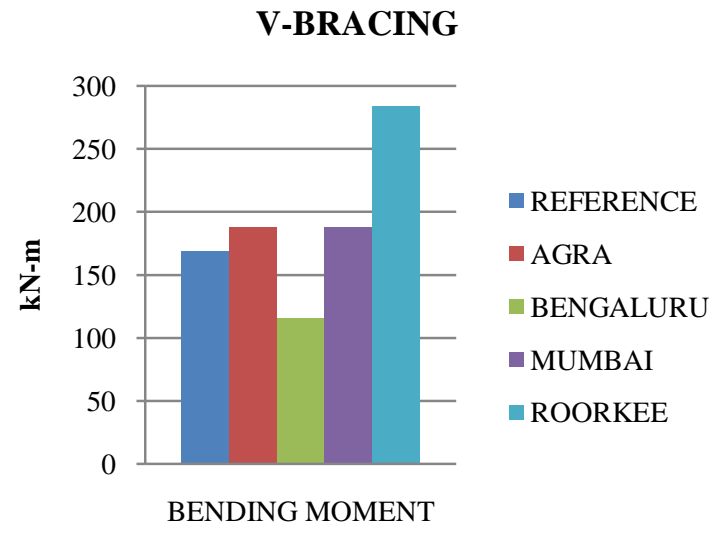

Fig. 27 Variation in Bending moment for different cities in $\mathrm{V}$ bracing 
Table 34. Maximum Bending moment acting on ground level column for $\mathrm{X}$ bracing system in $\mathrm{kN}-\mathrm{m}$

\begin{tabular}{|l|r|r|r|r|r|}
\hline & REFERENCE & AGRA & BLR & MUMBAI & ROORKEE \\
\hline BENDING MOMENT & 168.947 & 146.68 & 104.72 & 146.68 & 213.608 \\
\hline
\end{tabular}

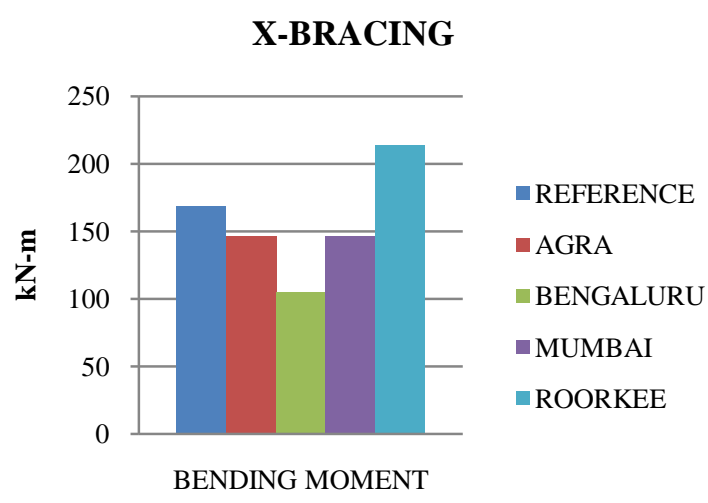

Fig. 28 Variation in Bending moment for different cities in $\mathrm{X}$ bracing

Table 35. Weight of steel in $\mathrm{kN}$ for various bracing systems in Agra

\begin{tabular}{|l|l|}
\hline Diagonal bracing & 9549.454 \\
\hline X bracing & 9727.191 \\
\hline V bracing & 9607.750 \\
\hline Knee bracing & 9635.323 \\
\hline Eccentric bracing & 9635.323 \\
\hline No bracing & 9635.323 \\
\hline
\end{tabular}

TAKE-OFF(AGRA)

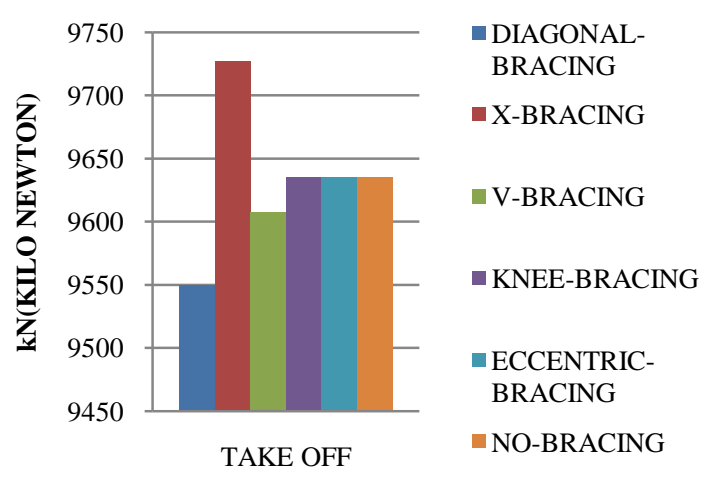

Fig. 29 Variation in Take-off for various bracing systems in Agra

Table 36. Weight of steel in $\mathrm{kN}$ for various bracing systems in Bengaluru

\begin{tabular}{|l|r|}
\hline Diagonal bracing & 9549.454 \\
\hline X bracing & 9727.191 \\
\hline V bracing & 9607.75 \\
\hline Knee bracing & 9635.323 \\
\hline Eccentric bracing & 9579.874 \\
\hline No bracing & 9635.323 \\
\hline
\end{tabular}

TAKE-OFF (BENGALURU)



Fig. 30 Variation in Take-off for various bracing systems in Bengaluru

Table 37. Weight of steel in $\mathrm{kN}$ for various bracing systems in Mumbai

\begin{tabular}{|l|r|}
\hline Diagonal bracing & 9549.454 \\
\hline X bracing & 9727.191 \\
\hline V bracing & 9607.75 \\
\hline Knee bracing & 9635.323 \\
\hline Eccentric bracing & 9579.874 \\
\hline No bracing & 9635.323 \\
\hline
\end{tabular}

TAKE-OFF(MUMBAI)

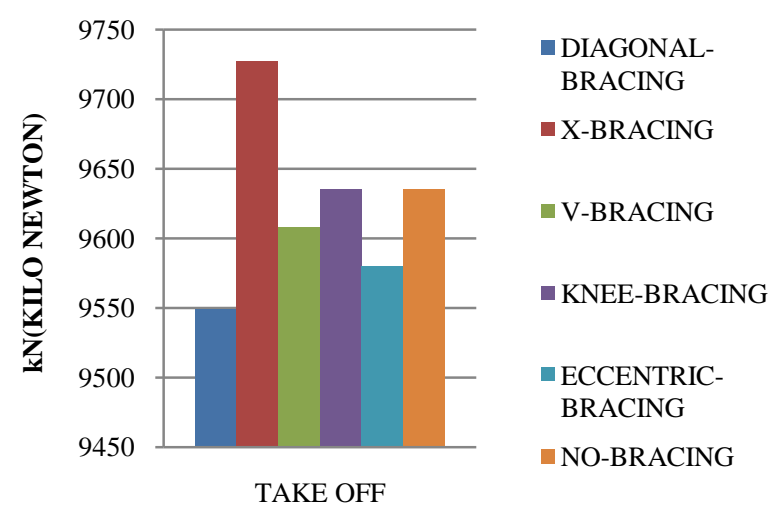

Fig. 31 Variation in Take-off for various bracing systems in Mumbai

Table 38. Weight of steel in $\mathrm{kN}$ for various bracing systems in Roorkee

\begin{tabular}{|l|r|}
\hline Diagonal bracing & 9549.454 \\
\hline X bracing & 9721.191 \\
\hline V bracing & 9607.75 \\
\hline Knee bracing & 9635.323 \\
\hline Eccentric bracing & 9579.874 \\
\hline No bracing & 9635.323 \\
\hline
\end{tabular}






Fig. 32 Variation in Take-off for various bracing systems in Roorkee

Table 39. Percentage change (\%) of drift index in Xdirection for Diagonal bracing

\begin{tabular}{|l|r|r|r|}
\hline Model & Displacement $(\mathrm{mm})$ & Drift index & Percentage change \\
\hline REFERENCE & 753.632 & 0.01621 & - \\
\hline AGRA & 660.092 & 0.01420 & 12.41 \\
\hline BENGALURU & 303.81 & 0.00653 & 59.69 \\
\hline MUMBAI & 485.587 & 0.01044 & 35.57 \\
\hline ROORKEE & 727.956 & 0.01565 & 3.41 \\
\hline
\end{tabular}

PERCENTAGE CHANGE IN X-DIRECTION



Fig. 33 Percentage change of drift index in $\mathrm{X}$ direction for Diagonal bracing

Table 40. Percentage change (\%) of drift index in Xdirection for Eccentric bracing

\begin{tabular}{|l|r|r|r|}
\hline Model & Displacement $(\mathrm{mm})$ & Drift index & Percentage change \\
\hline REFERENCE & 753.632 & 0.01621 & - \\
\hline AGRA & 692.317 & 0.01489 & 8.14 \\
\hline BENGALURU & 433.746 & 0.00933 & 42.45 \\
\hline MUMBAI & 692.317 & 0.01489 & 8.14 \\
\hline ROORKEE & 1039.125 & 0.02235 & 37.88 \\
\hline
\end{tabular}

\section{PERCENTAGE CHANGE IN X- DIRECTION}



Fig. 34 Percentage change of drift index in $\mathrm{X}$ direction for Eccentric bracing

Table 41. Percentage change (\%) of drift index in Xdirection for Knee bracing

\begin{tabular}{|l|r|r|r|}
\hline Model & Displacement(mm) & Drift index & Percentage change \\
\hline REFERENCE & 753.632 & 0.01621 & - \\
\hline AGRA & 686.475 & 0.01476 & 8.91 \\
\hline BENGALURU & 429.582 & 0.00924 & 43.00 \\
\hline MUMBAI & 686.475 & 0.01476 & 8.91 \\
\hline ROORKEE & 1028.998 & 0.02213 & 36.54 \\
\hline
\end{tabular}

\section{PERCENTAGE CHANGE IN X-} DIRECTION

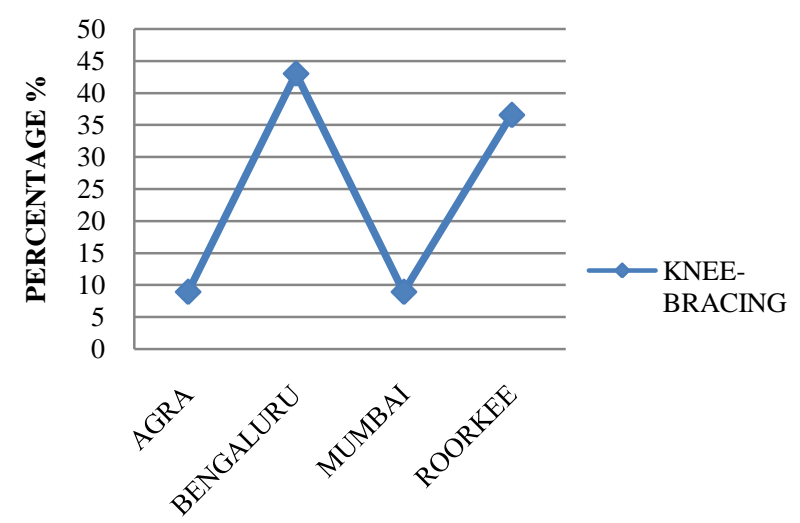

Fig. 35 Percentage change of drift index in $\mathrm{X}$ direction for Knee bracing

Table 42.Percentage change $(\%)$ of drift index in $\mathrm{X}$ direction for $\mathrm{V}$ bracing

\begin{tabular}{|l|r|r|r|}
\hline Model & Displacement $(\mathrm{mm})$ & Drift index & Percentage change \\
\hline REFERENCE & 753.632 & 0.01621 & - \\
\hline AGRA & 684.79 & 0.01473 & 9.13 \\
\hline BENGALURU & 428.617 & 0.00922 & 43.13 \\
\hline MUMBAI & 684.79 & 0.01473 & 9.13 \\
\hline ROORKEE & 1026.354 & 0.02207 & 36.19 \\
\hline
\end{tabular}




\section{PERCENTAGE CHANGE IN X- DIRECTION}

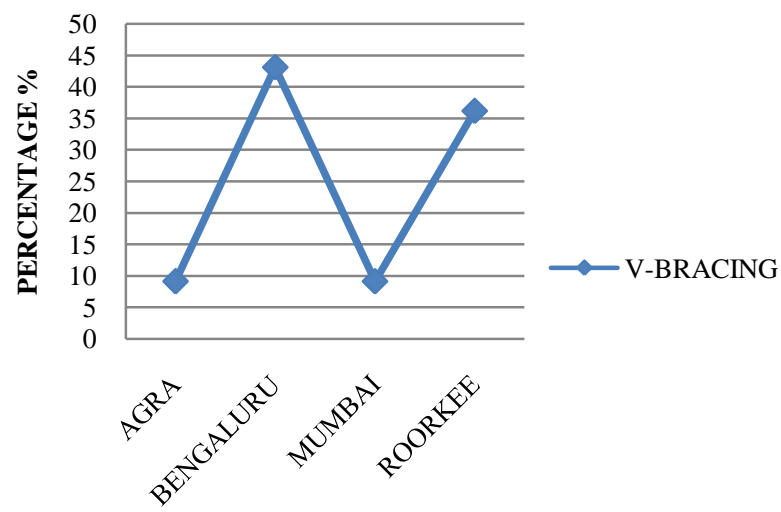

Fig. 36 Percentage change of drift index in $\mathrm{X}$ direction for $\mathrm{V}$ bracing

Table 43. Percentage change $(\%)$ of drift index in $\mathrm{X}$ direction for $\mathrm{X}$ bracing

\begin{tabular}{|l|r|r|r|}
\hline Model & Displacement $(\mathrm{mm})$ & Drift index & Percentage change \\
\hline REFERENCE & 753.632 & 0.01621 & - \\
\hline AGRA & 483.935 & 0.01041 & 35.79 \\
\hline BENGALURU & 302.811 & 0.00651 & 59.82 \\
\hline MUMBAI & 483.935 & 0.01041 & 35.79 \\
\hline ROORKEE & 725.434 & 0.01560 & 3.74 \\
\hline
\end{tabular}

PERCENTAGE CHANGE IN XDIRECTION

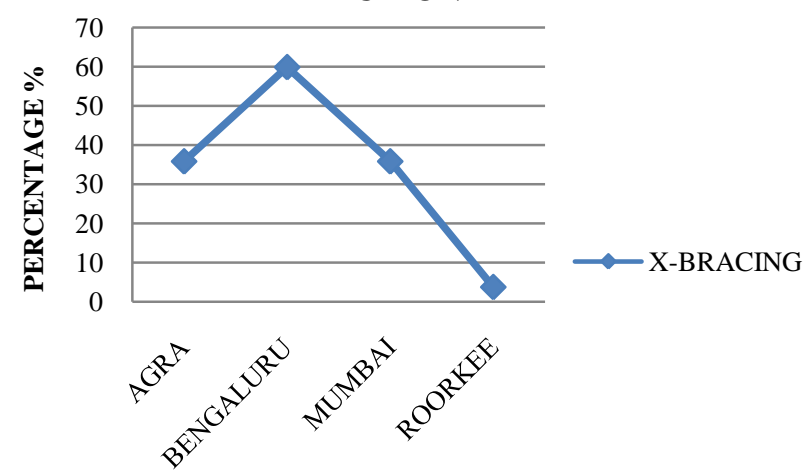

Fig. 37 Percentage change of drift index in $\mathrm{X}$ direction for $\mathrm{X}$ bracing

Table 44. Percentage change $(\%)$ of drift index in Zdirection for Diagonal bracing

\begin{tabular}{|l|r|r|r|}
\hline Model & Displacement $(\mathrm{mm})$ & Drift index & Percentage change \\
\hline REFERENCE & 1675.555 & 0.03603 & - \\
\hline AGRA & 1296.567 & 0.02788 & 22.62 \\
\hline BENGALURU & 597.112 & 0.01284 & 64.36 \\
\hline MUMBAI & 953.977 & 0.02052 & 43.07 \\
\hline ROORKEE & 1429.796 & 0.03075 & 14.67 \\
\hline
\end{tabular}

PERCENTAGE CHANGE IN ZDIRECTION



Fig. 38 Percentage change of drift index in $\mathrm{Z}$ direction for Diagonal bracing

Table 45. Percentage change (\%) of drift index in Zdirection for Eccentric bracing

\begin{tabular}{|l|r|r|r|}
\hline Model & Displacement(mm) & Drift index & Percentage change \\
\hline REFERENCE & 1675.555 & 0.03603 & - \\
\hline AGRA & 1397.031 & 0.03004 & 16.62 \\
\hline BENGALURU & 874.344 & 0.01880 & 47.82 \\
\hline MUMBAI & 1397.031 & 0.03004 & 16.62 \\
\hline ROORKEE & 2093.947 & 0.04503 & 24.97 \\
\hline
\end{tabular}

PERCENTAGE CHANGE IN ZDIRECTION



Fig. 39 Percentage change of drift index in $\mathrm{Z}$ direction for Eccentric bracing

Table 46. Percentage change (\%) of drift index in Zdirection for Knee bracing

\begin{tabular}{|l|r|r|r|}
\hline Model & Displacement $(\mathrm{mm})$ & Drift index & Percentage change \\
\hline REFERENCE & 1675.555 & 0.03603 & - \\
\hline AGRA & 1354.712 & 0.02913 & 19.15 \\
\hline BENGALURU & 847.919 & 0.01823 & 49.39 \\
\hline MUMBAI & 1354.712 & 0.02913 & 19.15 \\
\hline ROORKEE & 2030.437 & 0.04367 & 21.18 \\
\hline
\end{tabular}




\section{PERCENTAGE CHANGE IN Z- DIRECTION}



Fig. 40 Percentage change of drift index in $\mathrm{Z}$ direction for Knee bracing

Table 47. Percentage change (\%) of drift index in Zdirection for $\mathrm{V}$ bracing

\begin{tabular}{|l|r|r|r|}
\hline Model & Displacement $(\mathrm{mm})$ & Drift index & Percentage change \\
\hline REFERENCE & 1675.555 & 0.03603 & - \\
\hline AGRA & 1347.248 & 0.02897 & 19.59 \\
\hline BENGALURU & 843.509 & 0.01814 & 49.66 \\
\hline MUMBAI & 1347.248 & 0.02897 & 19.59 \\
\hline ROORKEE & 2018.901 & 0.04342 & 20.49 \\
\hline
\end{tabular}

\section{PERCENTAGE CHANGE IN Z- DIRECTION}

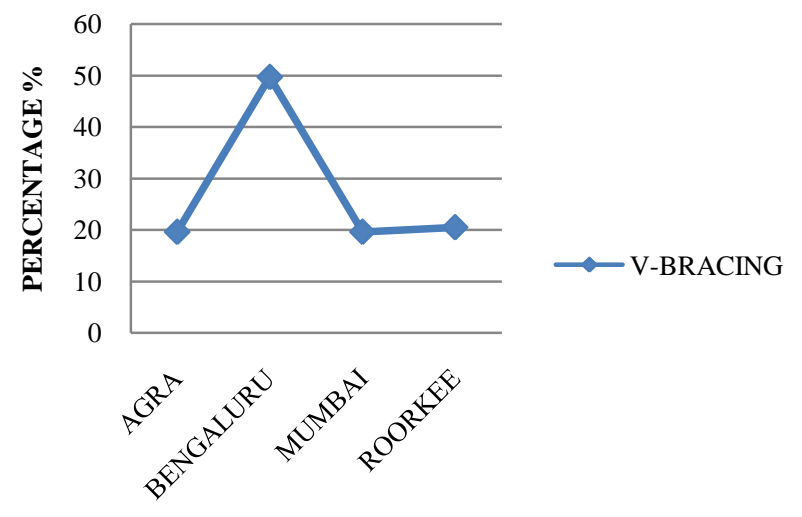

Fig. 41 Percentage change of drift index in $\mathrm{Z}$ direction for $\mathrm{V}$ bracing

Table 48. Percentage change $(\%)$ of drift index in Zdirection for $\mathrm{X}$ bracing

\begin{tabular}{|l|r|r|r|}
\hline Model & Displacement $(\mathrm{mm})$ & Drift index & Percentage change \\
\hline REFERENCE & 1675.555 & 0.03603 & - \\
\hline AGRA & 944.261 & 0.02031 & 43.64 \\
\hline BENGALURU & 591.174 & 0.01271 & 64.72 \\
\hline MUMBAI & 944.261 & 0.02031 & 43.64 \\
\hline ROORKEE & 1415.044 & 0.03043 & 15.55 \\
\hline
\end{tabular}

\section{PERCENTAGE CHANGE IN Z-} DIRECTION



Fig. 42 Percentage change of drift index in $\mathrm{Z}$ direction for $\mathrm{X}$ bracing

\section{CONCLUSION}

Following conclusions were drawn after analyzing the different bracing systems with un-braced reference model:

1. Bengaluru city has the least nodal displacements with respect to storey height in both $\mathrm{X}$ and $\mathrm{Z}$ directions from all the bracing systems compared to other cities.

2. Roorkee city has the highest nodal displacements with respect to storey height in Eccentric, Knee and V bracing system whereas; in Diagonal and $\mathrm{X}$ bracing systems the reference has the highest nodal displacements in both $\mathrm{X}$ and $\mathrm{Z}$ directions.

3. Mumbai and Agra cities show different nodal displacements with respect to storey height in Diagonal bracing whereas; it has same nodal displacements with respect to storey height on other bracing systems.

4. There is a $2.13 \%$ increase in axial force on reference model in Eccentric bracing in all cities.

5. Roorkee city shows the highest bending moment in all the bracing systems.

6. X bracing in all the cities shows the highest amount of steel required in $\mathrm{kN}$ whereas; Bengaluru city has the lowest amount of steel required in $\mathrm{kN}$.

7. Bengaluru city shows the highest percentage change in drift index in all the bracing systems in both $\mathrm{X}$ and $\mathrm{Z}$ directions.

\section{REFERENCES}

[1]. Narasimha Murthy K, Darshan SK, Karthik AS, Santosh R, Shiva Kumar KS, "Effective Study of Bracing Systems for Irregular Tall Steel Structures",International Journal of Scientific \& Engineering Research, Volume 7, Issue 5, may-2016 ISSN 2229-551.

[2]. Adithya. M , Swathi rani K.S , Shruthi H K, Dr. Ramesh B.R , "Study On Effective Bracing Systems for High Rise Steel Structures", SSRG International Journal of Civil Engineering, ISSN: 2348 - 8352, volume 2, Issue 2, February 2015, PP 19-22.

[3]. Praveena G.M and Thejaswini R.M,“Comparative Study of Different Bracing Systems for Irregular High Rise Steel Structure", International Journal for Scientific 
Research \& Development,ISSN (online): 2321-061, Vol. 3, Issue 09, 2015, PP 200-208.

[4]. Z.A. Siddiqi, Rashid Hameed, Usman Akmal, "Comparison of Different Bracing Systems for Tall Buildings", Pak. J. Engineering. \& Appl. Sci., Vol. 14, January 2014, PP 17-26.

\section{CODE PRACTICES}

[1]. IS: 800-2007, "General construction in steel code of practice" Third version, Bureau of Indian Standards, New Delhi, India.

[2]. IS: 1893 (Part 1)-2002, "Criteria for earthquake resistant design of structures" part 1 - general provisions and building, Fifth edition, Bureau of Indian Standards, New Delhi, India.

[3]. IS: 875 (Part 1,2,3)-1987, "Code of practice for design loads (other than earthquake) for building and structures, part 3 wind loads" 6th reprint, November 1998 Bureau of Indian Standards, New Delhi, India.

\section{BIOGRAPHIES}



Karthik K Vidyaranya, B.Tech Student Department of Civil Engineering, Global Academy of Technology, Bengaluru560098

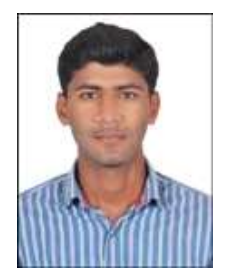

Shiva Kumar KS, Assistant Professor, Department of Civil Engineering, Global Academy of Technology, Bengaluru560098 